\title{
Disentangling coordination and alloy effects in transition metal nanoalloys from their electronic structure
}

\author{
L. Zosiak \\ Institut de Physique et Chimie des Matériaux de Strasbourg, \\ Université de Strasbourg, CNRS UMR 7504, \\ 23 rue du Lœss, BP 43, F-67034 Strasbourg, France and \\ M. Smoluchowski Institute of Physics, \\ Jagellonian University, Reymonta 4, 30-059 Krakow, Poland \\ C. Goyhenex \\ Institut de Physique et Chimie des Matériaux de Strasbourg, \\ Université de Strasbourg, CNRS UMR 7504, \\ 23 rue du Loess, BP 43, F-67034 Strasbourg, France \\ R. Kozubski \\ M. Smoluchowski Institute of Physics, \\ Jagellonian University, Reymonta 4, 30-059 Krakow, Poland \\ G. Tréglia \\ Centre Interdisciplinaire de Nanoscience de Marseille, \\ UPR 3118 CNRS, Campus de Luminy Case 913, \\ F-13288 Marseille Cedex 9, France
}

(Dated: June 21, 2013) 


\begin{abstract}
Accurate prediction of local properties of transition metal nanoalloys from the electronic structure is a challenge for building new materials with novel properties in a controlled way. To this aim, developping unified descriptions of local electronic states as a function of a minimal set of parameters is the way to disentangle structural and chemical effects. This is achieved here within $s p-d$ tight-binding calculations using a self-consistent procedure taking into account both the changes in the structural environment (coordination effect) and in chemical one (alloying effect). From these calculations, trends in the distributions of energy electronic states are obtained through band shifts and widths allowing one to study in a systematic way ordering tendency and local properties in nanoalloys in a wide range of sizes and structural complexity.
\end{abstract}

PACS numbers: 71.20.Be ; 71.23.An ; 71.15.Nc ; 64.70.kd; 64.75.Op 


\section{INTRODUCTION}

Owing to the recent development of nanoalloys [1, 2], within application fields like metallurgy, catalysis, magnetism and optics, a further challenge is to model alloying phenomena in nanosystems from the knowledge of the electronic structure of their pure constituents. This requires that the subtle coupling between electronic, structural and chemical effects is described and this understanding is used to build new structures with new properties. To this aim, many approaches have been developed, based upon a more or less sophisticated description of the electronic structure. Among them, ab initio DFT (Density Functional Theory)-type calculations [3-7], even the most performing[8], are too much time-consuming to allow systematic studies, whereas semi-empirical potentials[9-14] are too crude to establish accurately the link between the electronic, atomic and chemical structures. In this context, the Tight-Binding (TB) approximation [15, 16], which allows handling the electronic structure with a flexible accuracy depending on the addressed problem, is an intermediate suitable method for capturing the essential physics and will be used in the present work.

$s p-d$ TB calculations are performed in this paper by using a self-consistent procedure taking into account both the changes in structural environment (coordination effect) and in chemical one (alloying effect). The system chosen to illustrate the method is CoPt which presents a double interest. First it is archetypal of the class of ordering systems having a standard $d$-band shift behavior under alloying (decrease of diagonal disorder), as shown from XPS measurements [17] and calculations [18]. In addition, it is widely investigated for both potential applications in magnetic storage media and catalysis.

In this context the following work is dedicated to the determination of the two pertinent parameters characterizing order in an $\mathrm{AB}$ nanoalloy namely the diagonal disorder parameter $\delta_{d, 0}=\varepsilon_{d}^{A}-\varepsilon_{d}^{B}$ (difference between gravity centers of the d-bands, $\varepsilon_{d}$ ) and off-diagonal disorder parameter $\delta_{n d}=W_{d}^{A}-W_{d}^{B}$ (difference between d-band widths, $W_{d}$ ). These values have been shown to allow deriving ordering tendencies in all bulk transition metal alloys [19]. We intend here in addition to derive a law for the evolution of these parameters as a function of the size of clusters and to provide a tool for predicting ordering tendency in nanoalloys as a function of size which is an essential step for scientists willing to control the atomic arrangements 
in the fabrication of specific clusters for targeted properties. In a more applied frame, data like $\varepsilon_{d}$ should provide guidance to the interpretation of core level shifts measurements using X-Ray photoemission spectroscopy (XPS) in nanoalloys, a widely used technique in the field of catalysis for instance.

\section{TIGHT-BINDING METHODOLOGY: FROM ALLOYS TOWARDS NANOAL- LOYS}

For an alloy $A_{c} B_{1-c}$, the chemical configuration is defined from the set of site occupation factors $p_{i}^{a}$ such as $p_{i}^{a}=1$ if site $i$ is occupied by an atom of type $a(a=A, B)$ and $p_{i}^{a}=0$ otherwise. Then the corresponding hamiltonian is written in the basis of atomic orbitals $\lambda$ at sites $i,|i, \lambda\rangle$ :

$$
\begin{aligned}
H\left(p_{i}^{a}\right) & =\sum_{a=A, B} \sum_{i, \lambda} p_{i}^{a}|i, \lambda\rangle \varepsilon_{i \lambda}^{a}\langle i, \lambda| \\
& +\sum_{a, b=A, B} \sum_{i, j \neq i, \lambda, \mu \neq \lambda} p_{i}^{a} p_{j}^{b}|j, \mu\rangle\left(\beta_{i j}^{\lambda, \mu}\right)^{a b}\langle i, \lambda|
\end{aligned}
$$

which involves two types of parameters, the effective atomic levels $\varepsilon_{i \lambda}^{a}$ and the hopping integrals $\left(\beta_{i j}^{\lambda, \mu}\right)^{a b}$. In this framework $\varepsilon_{d}^{a}$ is the atomic $d$ orbital level for an atom of the type $a(a=A, B)$ in its own bulk and the hopping integral between two $d$-orbitals on neighboring sites drives the $d$ bandwidth $\left(W_{d}^{a}\right)[20]$. The first term gives rise to the so-called diagonal disorder effect coming from the difference $\delta_{d, 0}=\varepsilon_{d}^{A}-\varepsilon_{d}^{B}$ whereas the second one accounts for the possible effect of off-diagonal disorder due to the difference in $d$ bandwidth: $\delta_{n d}=W_{d}^{A}-W_{d}^{B}$. These two parameters $\delta_{d, 0}$ and $\delta_{n d}$ actually drive the redistribution of the electronic states with respect to those of pure elements and therefore both the new properties of the (nano)alloy and its preference for ordering or phase separation at low temperature. Based on these parameters, well known from compilations in the literature[21], systematic studies within the TB approach were previously carried out for deriving ordering tendencies in all bulk transition metal alloys [19] and general trends in $d$-band and core level shifts [18], the latter being tightly related e.g. to catalytic properties. The next step of the present work is to combine site coordination effects (atomic structure) and alloying effects (chemical structure) in a single model for nanoalloys in the experimental size range (up to thousands 
of atoms). The essential quantity to study is the local density of states (LDOS), $n(E)$. In all cases $s p-d$ hybridization is taken into account by using the basis of atomic orbitals $\lambda(s, p, d)$. Each partial LDOS $n_{i \lambda}(E)$ is obtained from the continued fraction expansion of the Green function $G(E)=(E-H)^{-1}[20,22,23]$ whose coefficients are directly related to the moments of the density of states. These coefficients are calculated within the recursion method[24] implemented with a self-consistent treatment of charge transfer induced by both coordination changes and alloying effects. The technique makes use of a local charge neutrality rule per site, per orbital and per chemical species, well known for surface effects and recently extended to alloys from DFT calculations [25] and further generalized for describing band shifts in bulk transition metal alloys[18]. Although of course some deviations from this neutrality rule shall be evidenced in some cases, also in experiments[26-28], our choice was to join a single law in order to bring the overall behavior of transition metal alloys under a single description which was indeed shown to be sufficient to describe d-band shifts and obtain a good agreement with photoemission experiments[18]. Here we propose a new application of this TB approach for nanoalloys, where both alloying and structural effects are included in the same procedure. In practice we consider ten pairs of exact coefficients in order to obtain sufficiently detailed LDOS. The main difficulty is then to determine the effective atomic level $\varepsilon_{i \lambda}$ for each inequivalent site $i$, while ensuring the charge self-consistency on this site. This requires, after indexing all inequivalent sites $i$ and species $a$, that these levels are shifted for each orbital $\lambda$ with respect to those in the bulk by a value $\delta \varepsilon_{i \lambda}^{a}$ following:

$$
\varepsilon_{i \lambda}^{a}=\varepsilon_{0 \lambda}^{a}+\delta \varepsilon_{i \lambda}^{a}
$$

in order to satisfy a given rule (here the local neutrality rule) on the different band fillings per orbital and per species $N_{i \lambda}^{a}$, which are obtained by integration of the partial local densities of states up to the Fermi level $E_{F}$ :

$$
N_{i \lambda}^{a}=\int_{-\infty}^{E_{F}} n_{i \lambda}^{a}(E) d E
$$

The total band filling (number of valence electrons) at site $i$, occupied by an atom of type $a$ is then determined by summing over all orbitals such as $N_{i}^{a}=\sum_{\lambda} N_{i \lambda}^{a}$.

The remaining parameters of the model are the hopping integrals between orbitals of 
neighboring sites, $\left(\beta_{i j}^{\lambda, \mu}\right)^{a b}$. If $a=b$ the values are directly taken from the compilation of D. A. Papaconstantopoulos[21] designed for elemental solids. For the specific case of Co, the parameters for the structural fcc paramagnetic phase were applied, in agreement with the fcc structure of the clusters considered hereafter. If $a \neq b$ the arithmetic average of $\beta^{\lambda, a}$ and $\beta^{\lambda, b}$ is used. Magnetism was not explicitly included in the TB calculations but some considerations about it will be given in the last section of this work.

The validity of this TB method must be supported by more fundamental ab initio calculations on the basic bulk phase alloys since the description of ordering behavior of complex nanoalloys is necessarily related to the bulk phase diagrams. We shall thereby verify the relative stability of the usual bulk alloy phases against possible competing ones. On the other hand the method must be proved to provide reliable local densities of states on which all interpretations in terms of local properties (as for instance band shifts for catalysis or density at Fermi level for magnetism) are related. For both purposes ab initio calculations within density functional theory (DFT) were performed using the SIESTA method[29]. SIESTA is based on the standard Kohn-Sham selfconsistent DFT which can be used either in the local-density (LDA) or in the generalized gradients (GGA) approximations for the electron-electron exchange and correlation interactions. The one-particle problem is solved using linear combination of atomic orbitals (LCAO) and taking as a solution method the diagonalization of the Hamiltonian. In the presented calculations we have used the generalized gradients (GGA) approximation of PBE[30] for the exchange and correlation potential. For the magnetic systems we performed spin-polarized calculations. For the ion-electron interactions, the core electrons are replaced by norm-conserving pseudopotentials [31]. Valence states are described using numerical atomic orbitals (NAO) and double- $\zeta$ polarized basis sets. The bulk lattice parameter was relaxed by a variable cell procedure within a conjugate gradient minimization. The low temperature experimental phase diagram of CoPt is characterized by three main ordered phases [32], the fcc $\mathrm{L}_{2}\left(\mathrm{Co}_{0.25} \mathrm{Pt}_{0.75}\right.$ and $\left.\mathrm{Co}_{0.75} \mathrm{Pt}_{0.25}\right)$ and the fct (tetragonalized with regards to fcc) $\mathrm{L} 1_{0}$ phase at equiconcentration. However, while considering in this work only the fcc crystalline structure, two ordered phases have to be considered at $\mathrm{c}=0.25$ concentration, in what is concerned relative stability, namely $\mathrm{L} 1_{2}$ $\left(\mathrm{Cu}_{3} \mathrm{Au}\right.$ type $)$ and also $\mathrm{DO}_{22}\left(\mathrm{Al}_{3} \mathrm{Ti}\right.$ type $)$. For $\mathrm{c}=0.5$ if $\mathrm{L}_{0}$ has to be the most stable it should be checked that the TB model well predicts this stability against other phases like 
the bcc $\mathrm{B}_{2}$ one. In this context we have calculated the relative stability energy $\Delta E_{\text {stab }}$ of the different ordered structures, at $\mathrm{c}=0.25$ and $\mathrm{c}=0.5$, both with DFT and TB methods. Within the latter only band energy, i.e the explicit term issuing from the electronic structure, has been considered. The results are presented in table I.

\begin{tabular}{c|c|c|c}
\hline Alloy & $\mathrm{Co}_{0.75} \mathrm{Pt}_{0.25}$ & $\mathrm{Co}_{0.5} \mathrm{Pt}_{0.5}$ & $\mathrm{Co}_{0.25} \mathrm{Pt}_{0.75}$ \\
\hline$\Delta E_{\text {stab }}$ & $E_{L 1_{2}}-E_{D O_{22}}$ & $E_{L 1_{0}}-E_{B_{2}}$ & $E_{L 1_{2}}-E_{D O_{22}}$ \\
Values in (eV/atom) & & & \\
\hline DFT (magnetic) & 0.001 & -0.247 & -0.007 \\
\hline DFT (non magnetic) & 0.003 & -0.337 & -0.012 \\
\hline TB (non magnetic) & 0.028 & -2.635 & -0.128 \\
\hline
\end{tabular}

TABLE I. Relative stability energy $\Delta E_{\text {stab }}(\mathrm{eV} / \mathrm{atom})$ between several bulk CoPt ordered alloys at different concentrations. DFT calculations were performed with (magnetic) and without (non magnetic) spin polarization.

There is a full consistency between DFT and TB concerning the sign of the obtained values of $\Delta E_{\text {stab }}$ and the hierarchy of the obtained relative energies. Absolute TB values of $\Delta E_{s t a b}$ are however different by about one order of magnitude than DFT ones. More surprising is the apparent degeneracy between the $\mathrm{L}_{2}$ and $\mathrm{DO}_{22}$ ordered structures obtained both in DFT and TB although the experimental phase diagram shows only the $L 1_{2}$ structure around $\mathrm{c}=0.25$ (or $\mathrm{c}=0.75$ ). However, it should be kept in mind that the structural difference between the $\mathrm{L}_{2}$ and $\mathrm{DO}_{22}$ consists only of the insertion of an antiphase boundary between two fcc unit cells leading to a periodic ordering on two fcc cells instead of one, making the two structures rather similar. Therefore the related energies of these two phases should not differ too much. This is deduced from a description of their density of states in terms of their moments, the DOS of the $\mathrm{L}_{2}$ and $\mathrm{DO}_{22}$ structures having six identical moments[33]. In the same sense, recent almost exhaustive calculations of bulk Co-Pt structures by R. V. Chepulskii and S. Curtarolo resulted in very close values for $\mathrm{Co}_{3} \mathrm{Pt} \mathrm{L1}_{2}$ and $\mathrm{DO}_{22}$ phases[34].

Then the reliability of the method for nanoalloys has been tested by making several DFT calculations on small clusters of different shapes and compositions and comparing the 
projected DOS with the LDOS obtained using the TB calculations. A good agreement is generally obtained, in this way validating the procedure of self-consistency based on a local neutrality rule per site, orbital and species. As an illustration we present in Fig.1 the result of such a conclusive comparison for a 147 atoms clusters showing a $\mathrm{L} 1_{0}$ order type with alternating Co and Pt (100) planes (see insert in Fig. 1).

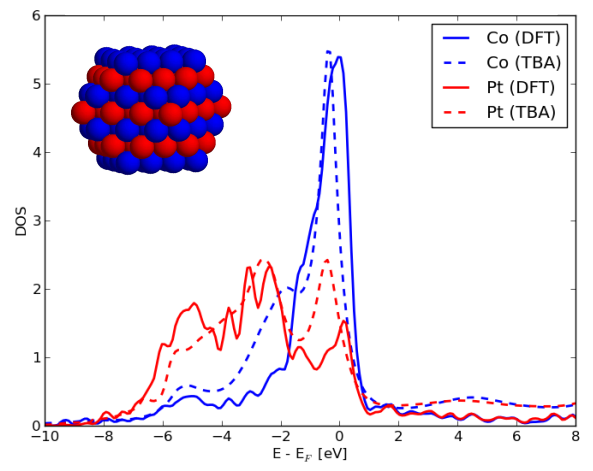

FIG. 1. (color on line) Averaged DOS (sp-d, non magnetic) in a cuboctahedral CoPt $L 1_{0}$-like cluster of 147 atoms. Solid lines correspond to DFT calculations and dashed ones to TB calculations. The insertion shows the corresponding atomic structure. Color code for both curves and structure in insertion is blue for Co and red for Pt.

Altogether, these comparisons establish well the reliability of our self-consistent $s p-d$ TB model in determining accurately the distribution of electronic states in nanoalloys and to a reasonnable extent its reliability to predict the relative stability between structures of different chemical arrangements.

\section{APPLICATION OF THE SELF-CONSISTENT SP-D TIGHT-BINDING METHOD TO COBALT-PLATINUM NANOALLOYS}

The usual shape for fcc clusters of sufficiently big size being the cuboctahedral one, we will build a model nanoalloy as a cuboctahedral piece of a bulk fcc $L 1_{0}$ structure. Such a system can be viewed as $n$ shell cuboctahedron presenting at its surface a sufficiently large set of inequivalent sites representative of realistic systems. Use of either Co or Pt centered clusters leads for the same size to variable concentrations $\left(0.46 \leqslant c_{a} \leqslant 0.54\right)$ and surface 
compositions. In order to differentiate chemical and geometrical effects, the knowledge of pure fcc Co and Pt clusters characteristics is obviously required. The resulting partial $d$ LDOS for all the inequivalent sites of both a pure Pt and a CoPt cluster, shown in Fig. 2, shows as expected an effective bandwidth which decreases with the site coordination (from facets to edges and vertices, see inset of Fig. 2.a) and is significantly modified near the Fermi level depending on the site. The values of the centers of gravity of the partial $d$-bands for the different inequivalent sites, $\varepsilon_{Z}^{P t}$ and $\varepsilon_{Z}^{C o}$, are plotted against their coordination numbers $Z$ in Fig. 3. Decreasing the size of a pure cuboctahedron from 2869 to 147 atoms does not bring much changes in $\varepsilon_{d}$ since the shape and, therefore, the local structural environment around each site remains unchanged. Regarding still pure systems, let us notice also the linear variation of $\varepsilon_{Z}^{P t}$ and $\varepsilon_{Z}^{C o}$ as a function of site coordination.
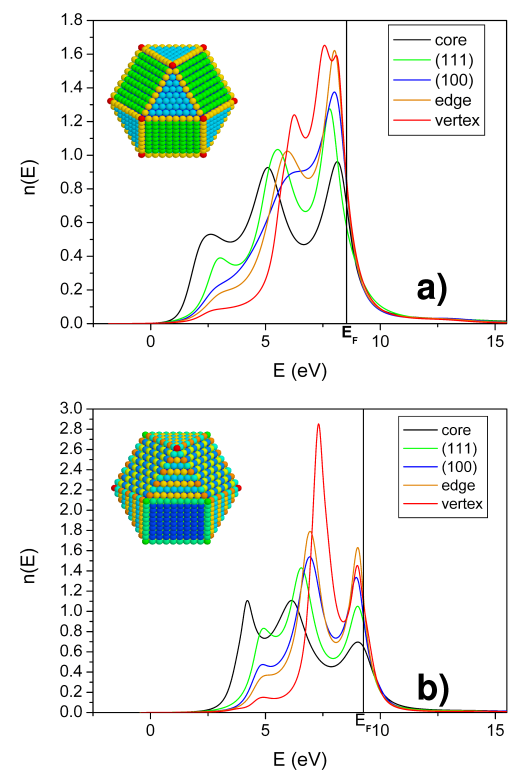

FIG. 2. (color on line) partial d-LDOS on the geometrically inequivalent sites on a cuboctahedral $\mathrm{Pt}$ (a) and CoPt $L 1_{0}$-like (b) cluster of 2869 atoms. The insertion shows the corresponding atomic structures, in which the sites have been colored upon their environment (coordination, chemical).

Comparing pure clusters to the mixed ones, the most remarkable feature is the evolution of the center of gravity of these bands which can be described in terms of geometry and alloying while disentangling both effects in a straightforward manner. Indeed the $Z$-variation of $\varepsilon_{d}$ for the alloy clusters is also nearly linear, though shifted in a rigid way from that in 

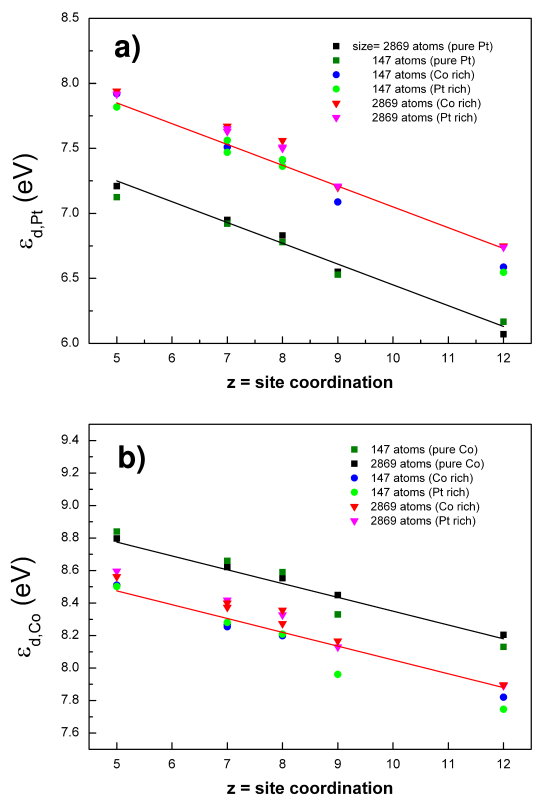

FIG. 3. (color on line) center of gravity of partial energy d-band for the geometrically inequivalent $\mathrm{Pt}$ (a) and Co (b) sites of cuboctahedral CoPt $L 1_{0}$ like clusters compared respectively to pure Pt and Co clusters. Two sizes are considered: $N_{t o t}=147$ and $N_{t o t}=2869$. Straight lines correspond to linear fits (see text). Label "Co rich" ("Pt rich") refers to concentration in Co (Pt) > 0.5.

pure clusters. Thus, the behavior of the atomic $d$-levels upon alloying in nanoclusters can be estimated by a linear variation law as a function of coordination $z$ to which an alloying term estimated by a rigid shift is added, which separates in an unambiguous way the structural and chemical effects. For $a=C o, P t$ the atomic $d$-level, $\varepsilon_{Z}^{a}$, is then written down as:

$$
\varepsilon_{Z}^{a}=\varepsilon_{0}^{a}+\Delta \varepsilon_{\text {alloy }}^{a}+\Delta \varepsilon_{\text {site }}^{a}(Z-12)
$$

The linear fit (see Fig. 3) leads to the following values of the constants: for the alloying term, $\Delta \varepsilon_{\text {alloy }}^{P t}=+0.6 \pm 0.15 \mathrm{eV}, \Delta \varepsilon_{\text {alloy }}^{\text {Co }}=-0.3 \pm 0.1 \mathrm{eV}$ and for the geometrical term, $\Delta \varepsilon_{\text {site }}^{P t}=-0.16 \mathrm{eV}, \Delta \varepsilon_{\text {site }}^{C o}=-0.085 \mathrm{eV}$.

A rather similar behavior is found in the case of the effective $d$-bandwidth $W_{Z}^{a}$, defined as the centered second moment of the LDOS for Z-coordinated sites, scaled by the same constant which relates this second moment to the actual bandwidth in the bulk. Thus a same linear equation can be derived just replacing $\varepsilon^{a}$ by $W^{a}$, with here $\Delta W_{\text {alloy }}^{P t}=-1.0 \pm 0.25 \mathrm{eV}$, 
$\Delta W_{\text {alloy }}^{C o}=+0.6 \pm 0.1 \mathrm{eV}, \Delta W_{\text {site }}^{P t}=-0.4 \mathrm{eV}$, and $\Delta W_{\text {site }}^{C o}=-0.22 \mathrm{eV}$. Further approximation of the alloying terms by the values of the actual bulk alloy, which is justified in view of our results, should allow a generalisation of these formulations to any bimetallic nanoalloy. Then, for a $n$ shells cuboctahedron, one is able to write an analytic formula for the dependence of the average atomic $d$-levels as a function of the cluster size :

$$
\varepsilon_{n}^{a}=\varepsilon_{0}^{a}+\Delta \varepsilon_{\text {alloy }}^{a}+\Delta \varepsilon_{\text {site }}^{a} \sum_{Z=5,7,8,9} x_{Z}^{a} \frac{N_{Z}}{N_{\text {tot }}}(Z-12)
$$

where $N_{\text {tot }}\left(=1+\frac{n}{3}\left(10 n^{2}+15 n+11\right)\right)$ is the total number of atoms, and the summation over $\mathrm{Z}$ means summing over the different under-coordinated sites, the respective numbers of which is $N_{Z}: N_{Z=5}=12$ vertices, $N_{Z=7}=24(n-1)$ edge atoms, $N_{Z=8}=6(n-1)^{2}$ atoms for the $(100)$ facet, $N_{Z=9}=4(n-1)(n-2)$ atoms for the $(111)$ facet. $x_{Z}^{a}=c_{Z}^{a} / c^{a}$ is the ratio between the partial $a$-concentration at a site of coordination $Z$ and $c^{a}$ the global $a$ concentration in the whole cluster. Hereagain, a similar equation can be used to describe the size-dependence of the effective $d$-bandwidth by just replacing $\varepsilon^{a}$ by $W^{a}$.

\section{ORDERING TRENDS IN NANOALLOYS}

From the results of the previous section, we are now able to return to the question of the ordering tendency in nanoalloys the ultimate goal being to be able to predict this tendency for any transition metal nanoalloy as a function of its size. Following the previous work on bulk alloy [19], the ordering tendency for a cluster of order $n$ will be linked to the differences $\delta_{d}^{n}=\varepsilon_{n}^{C o}-\varepsilon_{n}^{P t}$ and $\delta_{n d}^{n}=W_{n}^{C o}-W_{n}^{P t}$ obtained either by averaging all the Pt and Co LDOS in the cluster, respectively, or using the previously derived linear law. Averaging over all possible configurations in each case gives the law of variation for $\delta_{d}^{n}$ as a function of the size of the cluster, which writes analytically as:

$$
\begin{aligned}
\delta_{d}^{n} & =\delta_{d, 0}+\Delta \varepsilon_{\text {alloy }}^{\text {Co }}-\Delta \varepsilon_{\text {alloy }}^{P t} \\
& +\sum_{Z=5,7,8,9}\left(x_{Z}^{C o} \Delta \varepsilon_{\text {site }}^{C o}-x_{Z}^{P t} \Delta \varepsilon_{\text {site }}^{P t}\right) \frac{N_{Z}}{N_{\text {tot }}}(Z-12)
\end{aligned}
$$

This equation simplifies for a completely disordered alloy, or for a perfect $L 1_{0}$ system if one averages $C o$ and $P t$ centered clusters (as it is done here), since in these cases $x_{Z}^{a}=1$. 
One is then able to draw a diagram of this analytical law and to compare the resulting curve with the values obtained by averaging respectively all the Pt and Co LDOS in ordered clusters of different sizes. This is plotted in Fig. 4, in which we can see that bulk values are definitely reached around 2000 atoms (or in term of diameter as used in Fig. $4, N^{\frac{1}{3}} \approx 12$ ). The presented analytical model is based on approximations implying that only $d$-band shifts of the surface atoms are modified and where only prefactors are fitted to local values. Therefore an overall quantitative agreement with the values resulting from the LDOS averaging cannot be expected as it would be the case of an actual fit of these values. It is worth noticing that there is no basis to consider the increase of diagonal disorder parameter with the cluster size a general rule. Indeed the sign of the $Z$-dependent term in the equation (6) is driven by the difference $\Delta \varepsilon_{\text {site }}^{C o}-\Delta \varepsilon_{\text {site }}^{P t}$, which depends on the system under study, and can be deduced for any nanoalloy from the simple calculation of this dependence for both pure components. Finally a similar equation can be used to describe the $n$-dependence of the off-diagonal disorder parameter, replacing $\delta_{d}$ by $\delta_{n d}$ and $\varepsilon^{a}$ by $W^{a}$, the variation of which will also depend on the nature of the alloy through the sign of $\Delta W_{\text {site }}^{C o}-\Delta W_{\text {site }}^{P t}$.

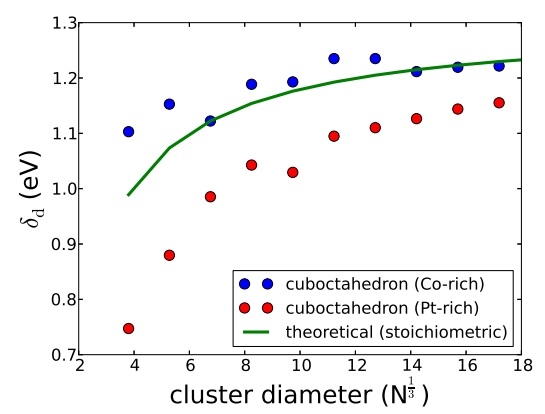

FIG. 4. (color on line) Variation of the diagonal disorder parameter in nanoalloys, $\delta_{d}^{n}$, as a function of $N^{\frac{1}{3}}$ (representative of the diameter of a cluster containing $N$ atoms). The continuous line represents the theoretical values given by equation (6). Red and blue circles represent values deduced from averaged LDOS respectively for the so-called $\mathrm{Pt}$ rich and Co rich ordered cuboctahedral clusters (see Fig.3).

Once the behavior of the two main parameters $\left(\delta_{d}^{n}, \delta_{n d}^{n}\right)$ which drive the electronic structure of a nanoalloy of size $n$ is known, let us show how the mapping previously derived to predict ordering tendency for bulk alloys [19] can be extended to the case of nanoalloys. A 
relevant way to visualize the alloying effects in clusters $v s$ bulk effects is to build a mapping of ordering and demixing domains in the parameter space spanned by $\delta_{n d}$ and $d$-band electronic filling $N_{e}$ for different values of $\delta_{d}$ and for the concentration of interest, here $c=0.5$. Results are presented in Fig. 5. In contrary to Ref. [19], here we focus on a single set of values corresponding to an average value of $d$-bandwidth related to CoPt and equal to $6 \mathrm{eV}$. The most significant effect is that for $\delta_{d}=2.0 \mathrm{eV}$ (black curve) only one large and centered domain appears meaning in particular that the corresponding alloys with $N_{e}>8$ and $N_{e}<2$ should show strictly demixing tendency. However reducing $\delta_{d}$ to at least $1.0 \mathrm{eV}$ reveals new ordering domains at higher electronic filling allowing again order tendency for late transition metal alloys. It remains to introduce the cluster points in this bulk map, by postulating that it can be done by just moving the bulk point according to the values of $\delta_{d}^{n}, \delta_{n d}^{n}$ issued from the previous laws. In addition, consistently with the charge neutrality rule, these points will be always positioned at the electronic filling of $N_{e}=8.5$ characteristic of $\mathrm{CoPt}$ at equiconcentration. In order to cover both possible size and morphological effects we have considered two limiting sizes (147 and 2869 atoms) and two morphologies adding icosahedra to the previously investigated cuboctahedra. As can be seen from the the corresponding specific points in Fig. 5, all the systems fall at the frontier of the upper ordering domains.

A general behavior of the evolution of electronic structure in CoPt nanoalloys can be tentatively deduced from these results. Actually, a global evolution is obtained when going from bulk alloy to clusters with a large decrease of the absolute value of off-diagonal disorder. Further decrease of diagonal disorder is found when decreasing the size of the clusters from 2869 atoms to 147 atoms. Since icosahedral and cuboctahedral shapes are leading to similar results it can be concluded that the structure has a weak effect on the ordering tendency. It is also worth noticing that even though the points are not falling directly in the upper ordering domains their displacements as a function of size strictly follows the shift towards lower absolute values of $\delta_{n d}$, indicating that order tendency does not change for any $\mathrm{CoPt}$ nanoalloy. The possible role of magnetism was not yet invoked although, as recently proposed by S. Karoui and coworkers [35], in a spin polarized description of the CoPt LDOS, the majority spin up band is completely full and does not participate to the cohesion of the alloy so that an effective average electronic filling of $6.6 d$ electrons should be attributed to 
CoPt systems. In this case all the points of Fig. 5 would be shifted down into the larger ordering domains, meaning that once again the bulk ordering tendency is not only preserved but even enhanced in the cluster.

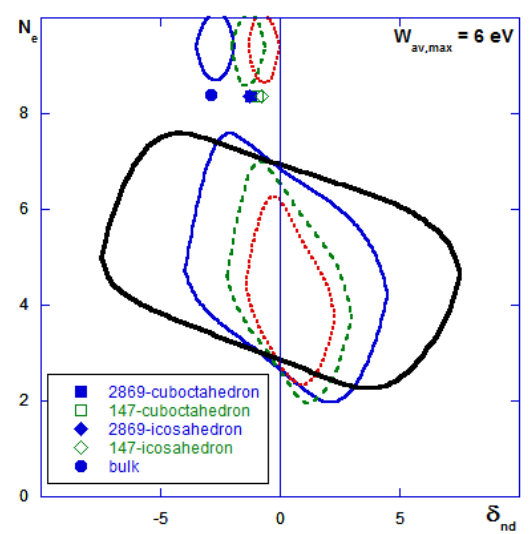

FIG. 5. (color on line). $\left(N_{e}, \delta_{n d}\right)$ ordering domains represented in the parameter space spanned by $\delta_{n d}$ and $N_{e}$ for different values of $\delta_{d}$ and for alloys at equiconcentration. Ordering domains are delimited by black, blue, dotted green and dotted red lines corresponding respectively to $\delta_{d}=2.0$, 1.0, 0.5, $0.25 \mathrm{eV}$, the area outside these domains corresponding to demixing tendency. This plot is related to an average bandwidth of pure constituents of $W_{a v, \max }=6.0 \mathrm{eV}$. The points correspond to bulk and 2869 and 147 atoms clusters.

\section{CONCLUSION}

In summary, we have presented an accurate way to describe and predict the main local characteristics of nanoalloys, namely their mixing behavior and redistribution of energy electronic states from the single knowledge of the electronic structure of their pure constituents, using a self-consistent TB approach in the direct space. Local effects can be quantified through linear laws as a function of site coordination, by disentangling structural and chemical effects in a straightforward manner. Such a methodology is extendable to any other alloy since it has been shown to give a unified description of local electronic structure both at surfaces of pure materials and in pure bulk alloys. Thus, analytic laws have been derived which give the variation of both $d$ atomic level and effective $d$ bandwidth on one hand, diagonal and off-diagonal disorder parameters on the other hand, as a function of 
cluster size. Ordering trends in nanoalloys can then be estimated from bulk stability maps by just shifting the corresponding $\left(\delta_{d}, \delta_{n d}\right)$ points according to these analytic laws. This method has no limit neither in size, nor in structural asymmetric features, allowing the presence of surfaces, low coordinates sites, defects like vacancies or stacking faults.

Acknowledgments. This work was founded by the French National Agency ANR through ETNAA Project No. ANR-07-NANO- 018 and the Hubert Curien Program PHC Polonium No. 22556UC. We thank the "Institut du Développement et des Ressources en Informatique Scientifique" (IDRIS) for a grant of computer time (Project No. 92291). Fruitful discussions with H. Amara, F. Ducastelle and B. Legrand are gratefully acknowledged.

[1] R.L. Johnston and R. Ferrando, Nanoalloys: from theory to application, Faraday Discussions 1389 (2008).

[2] Nanoalloys: Synthesis, Structure and Properties, D. Alloyeau, C. Mottet and C. Ricolleau, (Eds), Springer London (2012).

[3] G.H. Johannesson, T. Bligaard, A. V. Ruban, H. L. Skriver, K. W. Jacobsen and J. K. Norskov, Phys. Rev. Lett. 88255506 (2002).

[4] A.V. Ruban, S. Shallcross, S.I. Simak, and H. L. Skriver, Phys. Rev. B 70125115 (2004).

[5] A.V. Ruban and I.A. Abrikosov, Rep. Prog. Phys. 71, 046501 (2008).

[6] L.G. Ferreira, V. Ozolins and A.Zunger Phys. Rev. B 60, 1687 (1999).

[7] G. Ghosh, A. van de Walle and M. Asta, Acta Mater. 56, 3202 (2008).

[8] M. E. Gruner, G. Rollmann, P. Entel, and M. Farle, Phys. Rev. Lett. 100, 087203 (2008).

[9] S.M. Foiles, M.I. Baskes and M.S. Daw, Phys. Rev. B 337983 (1986).

[10] K.W. Jacobsen, O. Stoltze, J.K. Norskov, Surface Science 366394 (1996).

[11] M.W. Finis and J.E. Sinclair, Phil. Mag. A50, 45 (1984).

[12] V.Rosato, M.Guillopé, and B.Legrand, Phil. Mag. A59, 321 (1989).

[13] A.P. Sutton and J. Chen, Phil. Mag. Lett. 61, 139 (1990).

[14] G.J. Ackland and S.K. Reed, Phys.Rev. B 67, 174108 (2003).

[15] see all contributions in Comput. Mat. Science 12 157-287 (1998). 
[16] Tight-Binding Approach to Computational Materials Science, Mat. Res. Soc. Symp. Proc. 491 Eds. P. Turchi, A. Gonis, Colombo,(1998).

[17] Y-S. Lee, K- Y. Lim, Y- D. Chung, C- N. Wang, and Y. Jeon, Surf. Interface Anal. 30, 475 (2000).

[18] C. Goyhenex and G. Tréglia, Phys. Rev. B 83, 075101 (2011).

[19] J.H. Los, C. Mottet, G. Tréglia, and C. Goyhenex Phys. Rev. B 84, 180202 (R) (2011).

[20] F. Ducastelle, Order and Phase Stability in Alloys (North Holland, 1991).

[21] D.A. Papaconstantopoulos, Handbook of Electronic Structure of elemental Solids Plenum, New York, (1986).

[22] M. C. Desjonquères and D. Spanjaard, Concepts in Surface Science, Springer, 1995.

[23] G. Allan and M. Lannoo, J. Phys. Chem. Solids 37, 699 (1976).

[24] R. Haydock, V. Keine, and M. J. Kelly, J. Phys. C 8, 2592 (1975).

[25] A. Jaafar, C. Goyhenex, and G. Tréglia, J. Phys. Condens. Matter 22, 505503 (2010).

[26] R. J. Cole, N. J. Brooks, and P. Weightman, Phys. Rev. B 56, 12178 (1997).

[27] P. A. Korzhavyi, A. V. Ruban, I. A. Abrikosov, and H. L. Skriver, Phys. Rev. B 51, 5773 (1995).

[28] Y. S. Lee, J. Y. Rhee, C. N. Whang, and Y. P. Lee, Phys. Rev. B 68, 235111 (2003).

[29] J. M. Soler, E. artacho, J. D. Gale, A. Garcia, J. Junquera, P. Ordejon and D. Sanchez-Portal, J. Phys.: Condens. Matter 14, 2745 (2002).

[30] J. P. Perdew, K. Burke and M. Ernzerhof, Phys. Rev. Lett. 77, 3865 (1996).

[31] N. Troullier and J. L. Martins, Phys. Rev. B 43, 1993 (1991).

[32] C. Leroux, M. C. Cadeville, V. Pierron-Bohnes, G. Inden, F. Hinz, J. Phys. F: Met. Phys. 18, 2033 (1988).

[33] A. Bieber, F. Ducastelle, F. Gautier, G. Tréglia and P. Turchi, Solid State Commun. 45, 585 (1983).

[34] R. V. Chepulskii, and S. Curtarolo, Appl. Phys. Lett. 99, 261902 (2011).

[35] S. Karoui, H. Amara, B. Legrand and F. Ducastelle, J. Phys.: Condens. Matter 25, 056005 (2013). 